\title{
WHITE DWARFS
}

\section{Follow the shock front}

Nature https://doi.org/10.1038/s41586-020-

2535-y (2020)

Most stars, including the Sun, will end up as white dwarfs. With roughly half a solar mass crammed into an Earth-sized object, the resulting high density of matter means that the electrons are forced into a degenerate gas of closest packed states. They cannot be further compressed, and it's this electron pressure that prevents further gravitational crunching. As such pressures are hard to attain in the laboratory, stellar models of the interior structure of white dwarfs lack stringent tests in this regime. Andrea Kritcher and co-workers now report experiments on a hydrocarbon $\left(\mathrm{C}_{9} \mathrm{H}_{10}\right)$ at up to 450 million atmospheres. They apply their measurements of the equation of state to pulsating white dwarfs with carbon-dominated atmospheres (known as hot DQ stars).

The experiments at the National Ignition Facility in Livermore, California, use high-powered lasers to create an X-ray radiation bath inside a gold cylinder. An ablator heats and expands, launching shock waves towards a central sphere of $\mathrm{C}_{9} \mathrm{H}_{10}$. The density, pressure, speed and opacity at the shock front are all recorded. Kritcher et al. were able to measure the pressure-density curve of the shock Hugoniot of $\mathrm{C}_{9} \mathrm{H}_{10}$, finding that the shape of the Hugoniot is consistent with models that account for the electronic shell structure of the carbon core electrons and predict an increase in compressibility due to ionization of the inner core orbitals. These insights will help to refine interior models of these pulsating stars, which will in turn benefit from higher pressure measurements in the future.

May Chiao

Published online: 10 August 2020

https://doi.org/10.1038/s41550-020-1188-y 